\title{
Determination of early bone metastasis on Bone Scans Using the Gray Levels Histogram
}

\author{
Búsqueda de metástasis ósea temprana en gammagramas óseos usando \\ el histograma de tonos de gris
}

\author{
M. Pérez-Meza'; A. Jaramillo-Núñez²; B. Cuevas-Otahola²; J. A. Arriaga-Hernández³; B. E. Sánchez-Rinza \\ 'Licenciatura en Informática, Universidad de la Sierra Sur \\ ${ }^{2}$ Coordinación de Óptica, Instituto Nacional de Astrofísica, Óptica y Electrónica \\ ${ }^{3}$ Facultad de Ciencias Fisicomatemáticas, Benemérita Universidad Autónoma de Puebla \\ ${ }^{4}$ Facultad de Ciencias de la Computación, Benemérita Universidad Autónoma de Puebla
}

\section{ABSTRACT}

The aim of this paper is to show a technique to speed up the interpretation of bone scans in order to determine the presence of early bone metastasis. This is done using the gray levels histogram of the region of interest. The technique is intended to assist in the bone scans interpretation in order to provide a successful diagnosis. During the analysis, three types of histograms were observed on the regions of interest. If the histogram is narrow and shifted toward the origin, the bone scan is free of metastasis. If it is shifted to the right and slightly broadened, indicates the presence of a bone anomaly different from a metastasis. On the other hand, if the histogram is more broadened and shifted to the right, is suggests the presence of metastasis. This histogram is characterized by displaying small curls on the right side providing information about the metastatic disease stage, which could be low-amplitude peaks and have a short length, if the metastasis is in early stage, or high-amplitude peaks and a long length, if is advanced. Finally, the analyzed region is displayed in false color considering the minimum gray levels observed in the histogram.

KEYWORDS: Bone scan; skeletal metastasis; image histogram; digital image processing

\section{Corresponding author}

TO: Mónica Pérez Meza

INSTITUTION: Universidad de la Sierra Sur

ADDRESS: Guillermo Rojas Mijangos S/N, esquina con

Avenida Universidad, A. P. 70800, Miahuatlán de

Porfirio Díaz, Oaxaca, México

E-MAIL: arielmonp@hotmail.com

\section{Received:}

24 September 2020

Accepted:

16 December 2020 


\section{INTRODUCTION}

Among malignant neoplasms, prostate cancer has a considerably high incidence rate in 65-year-old men and older. For instance, 230000 new cases were diagnosed in the US in $2005^{[1]}$. In 2018, between 21000 and 25000 new cases were diagnosed in Mexico, representing $11.7 \%$ of the total cancer-detected cases. From this total approximately 7000 patients died during the course of the year ${ }^{[2]}$. The main prostate cancer risk factors are age, race, as well as genetics (if a first-grade relative suffers the disease, the probability increases by a factor of 2). Hence, an objective of the health system is the early detection using screening strategies, through the use of specific prostatic antigen, focused in 50-year-old men and above.

The bone is third most common location likely to develop metastatic disease, below the lungs and liver [3]. It is well known that once the patient has been diagnosed with metastatic disease, the prognosis of life expectancy is short, due to the dissemination of the disease to the bones, being unlikely to be cured. However, in each case, available treatments can be used to retard the disease advance, and increase the life expectancy.

The patients' knowledge of such a treatment is essential for the treatments choice. Thus, the purpose of imaging techniques is to identify the early phases of an ongoing bone disease, in order to determine its extension, and subsequently address the possible complications, such as pain, pathological fractures, hypercalcemia or medullary compression. Also, it is remarkably useful in evaluating the response to a treatment, serving as a guide during a biopsy procedure to obtain a diagnostic confirmation of the disease.

Diagnosis of bone metastases through imaging techniques basically consists of direct visualization of tumor infiltration or detection of bone reaction to the tumor process.
Bone scintigraphy is the most common imaging modality used to evaluate cancer-to-bone dispersion. In most cancer centers their interpretation is performed visually, however, to give successful diagnoses a vast experience is required due to the difficulties associated with the recognition of hot spots (areas with high marker content). Hence, a quantitative rather than a qualitative interpretation would be more useful in the bone scans interpretation to improve and standardize diagnoses ${ }^{[4]}$.

\section{Background}

Several computer-assisted methods have been developed to find metastasis. Some techniques use the bone scan index ${ }^{[5]}$, neural networks ${ }^{[6]}$ and false color ${ }^{[7]}$. All of them require a large number of bone scans datasets to calibrate the system, resulting in time-consuming procedures.

In this work we introduce a method based on the patients' bone scans analysis, intended to determine the presence of metastatic disease. To this aim a segmentation procedure is required, which is the first step. Subsequently, the gray levels histogram of the region of interest (ROI) is displayed and observed. The image segmentation is required for performing the analysis by region due to the diverse bone densities and their probability to develop a metastatic disease. The diagnosis of bone metastasis using image processing techniques is based on the direct visualization of tumor infiltration or bone reaction to metastatic disease.

Bone scans images are often stored in an archive, where a pixel can contain up to 1024 values (210 umbers, bit depth). In the case of a healthy skull, free of bone diseases, we observed that its gray tones did not reach values above 60 . The same behavior holds for healthy bones ${ }^{[8]}$. There are only two regions where the gray levels could reach the value 1024: the zone where the radiotracer is injected and the bladder, which is where the unsorted marker is stored. 
However, these regions do not matter for diagnosis, given their high values, being inconclusive to detect metastasis. Throughout the whole analysis and visualization data is always used in DICOM format ("raw" DICOM images).

In the case of a bone free of metastasis, the image gray levels interval is small, of the order of 60 tones. This interval is larger in the presence of a bone anomaly.

The main objective of the method presented in this work is to detect early bone metastasis. The specialist should draw special attention to the gray levels of the regions of interest (ROI) with values less than 80. Gray tones above such value correspond to advanced bone metastasis, easily observable in the bone scan, so these images don't matter to the method.

Histograms actually obtained from the ROI's are of bimodal type, in such a way that the determination of a malignant bone abnormality will be based mainly on the observation of the right end of the histogram of the ROI.

\section{MATERIALS AND METHODS}

An observational, retrospective, and analytic study was conducted in the Nuclear Medicine Department of the National Medical Center "La Raza" at IMSS [7]. The sample consists 138 patients diagnosed with prostate cancer with ages ranging from 47 to 85 years. The detection was performed in bone gammagrams following the Gleason classification. The gammagrams were captured after giving an intravenous dose of $25 \mathrm{mCi}$ de 99mTc-MDP and 2 litters of water to the patients. The whole-body scans were performed two hours after giving the patients the radiopharmaceutical, using a dualhead MEDISO INTERVIEW XP VERSION 1.05014 with a LEHR collimator with a velocity of $12 \mathrm{~cm} / \mathrm{min}$ in anterior and posterior projections. The bone gammagrams were subsequently visually inspected by three specialists in the Nuclear Medicine Department, inde- pendently. We used the obtained diagnoses as a reference during this research. Out of the 138 bone gammagrams, 43 were of the whole-body type, constituting the sample used for our purposes.

In the context of image processing, image segmentation is among the most intricate techniques. It is used to split a given image into several sub-images having common features. The main goal of such a technique is to simplify the image representation in terms of meaningful data. There are two types of image segmentation: local (concerning to separate image regions) and global (concerning to the segmentation procedure of a complete image formed by a large pixel number).

The local image segmentation is the main scope of this research. Such procedure is considered as part of the developed software along with the previously mentioned techniques. Image segmentation using the histogram-based thresholding procedure is probably the most common approach, since it is easy to implement and requires less computational resources to be executed. These methods generally employ the maximization or minimization of a criterion function based on the image histogram. The optimal threshold is the gray level intensity at which the criterion function reaches its maximum or minimum values. In our case we use the minimum value.

Several methods for image segmentation are available in the literature. We used the variance between classes method (VBC), in this work, to find the minimum value between two Gaussian distributions. Such a method uses a discriminant function to determine the optimal threshold of an image histogram, in order to perform the image segmentation in near uniform regions ${ }^{[9]}$.

In some cases, it is required to perform the segmentation procedure in tri-modal histograms, hence two thresholds are required. An iterative algorithm based 
on the maximization of the VBC was proposed in Reddi, Rudin and Keshavan ${ }^{[10]}$. We will refer to these images as tri-modal images.

The algorithms used in this work were developed in Matlab. These algorithms determine one or two thresholds, depending on each case, similar to the procedures in the algorithms developed by Demirkaya et al ${ }^{[11]}$. For the two-thresholds case, an iterative implementation for the VBC method was used.

We display the segmented ROI in color, and we regard the region edges as level contours or curves since pixels with equal gray tones form a contour.

\section{Development}

Our proposed software splits the bone scan in six ROIs: skull, shoulders, thorax, vertebral spine, scapula and pelvis. Such a segmentation is performed considering the higher probabilities of the bones in these regions to develop a metastatic disease.

Subsequently, the split image histogram is displayed, showing its bi-modality. The first global histogram minimum indicates the gray tone separating the ROI image background, which will refer to as min. When the ROI is constituted by a metastasis-free bone, the right end of the histogram drops to zero quickly. We will refer to this minimum as "max". However, if the ROI contains a metastasis-free bone, with another pathology or disease, the histogram will broaden in the presence of a degenerative disease, such as osteoporosis, osteopenia, etc.

On the other hand, if the ROI shows signs of metastatic disease, the right end of the histogram will approach zero slowly and monotonically, showing small lobes (local maxima with low amplitude). The lobes extension will depend on the metastatic disease stage. Early metastatic disease is characterized by small-amplitude and short-extension lobes.
In order to illustrate this method, we show the analysis of the skull and pelvis ROIs (the segmentation procedure was performed in the whole-body scan using the previously mentioned techniques). We show only two regions since the rest of the regions show a similar behavior.

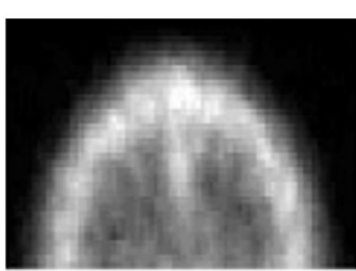

(a)

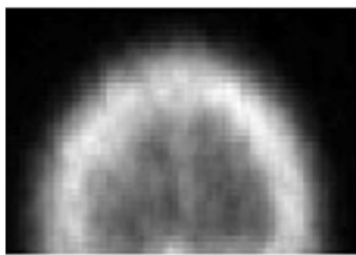

(c)

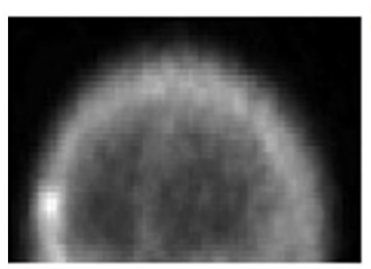

(e) (b)

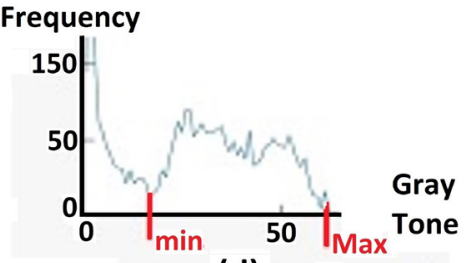

(d)

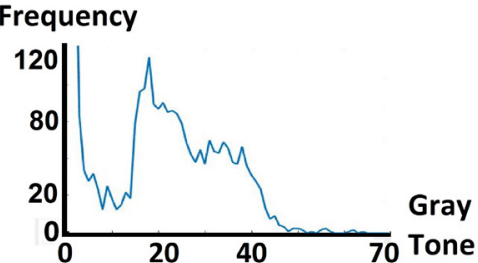

(f)

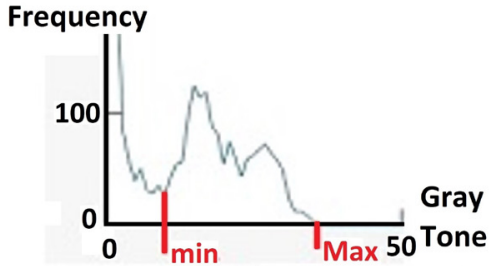

FIGURE 1. ROI images of the skull. In (a) healthy case, in (c) degenerative disease, in (e) metastatic disease. The corresponding histograms of (a), (c) and (e), are shown in (b), (d) and (f), respectively.

If the region is embedded in a dark background, as in the skull case, the histogram will be similar to the one shown in Figure 1.a. The minimum gray tone is shown by a red line in the left of the plot, separating the dark background from the ROI. In the case of healthy skull, the maximum gray tone value will be below 50 . Moreover, the histogram will quickly approach to zero. In the presence of a degenerative disease the max value will be above 50 and the plot width will increase, as in Figure 1d. The histogram end will approach to zero in descending form, shifted to the right regarding the healthy body histogram. 
The most relevant case corresponds to that showing an early-stage metastasis, which is analyzed in the histogram in Figure 1f. In the figure the gray tones corresponding to such a case are beyond the ending region of the healthy-body histogram (beyond the gray tone with value 50), shown as small variations, extending along the gray tone axis.

The pelvis bone probability to develop degenerative diseases as well as metastasis, is high. Hence, the corresponding histograms are similar the previous case. The skull ROI, displays as well a high probability of developing several types of pathology, implying that the different pathology cases (including pathology types different than cancer) have similar histograms, as seen in Figure 2.

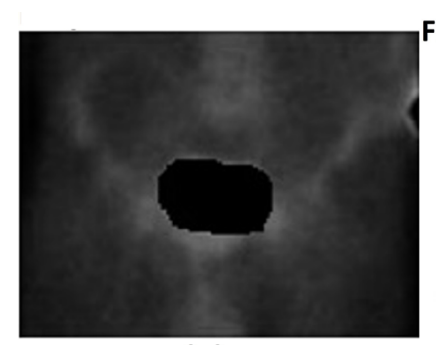

(a)

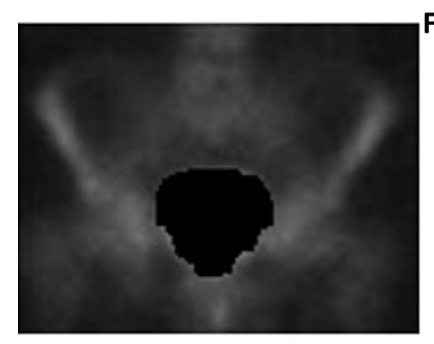

(c)

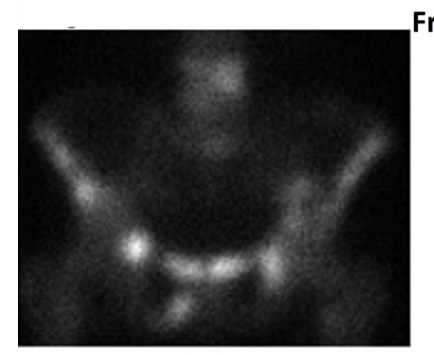

(e)

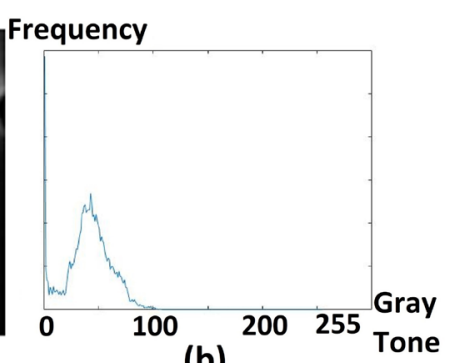

(b)

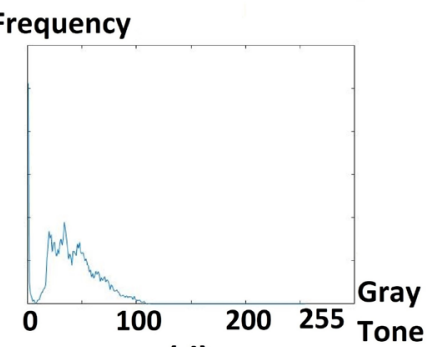

(d)

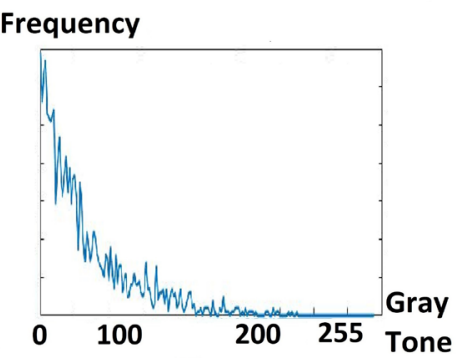

(f)
FIGURE 2. ROI images of the pelvis. In (a) healthy case, in (c) degenerative disease, in (e) metastatic disease. The corresponding histograms of (a), (c) and (e), are shown in (b), (d) and (f), respectively.
Such a knowledge can be obtained by the specialist from training devoted to study the bone scans histograms. On the other hand, the software includes a visualization tool to display the analyzed ROI's using false color. Such a tool adds color from a list of seven colors assigned to gray levels intervals, setting as a reference the min and max observed values obtained from the histogram analysis. The color assignation scheme is summarized in Table 1.

\section{TABLE 1. Assigned color intervals in terms of the min and max values.}

\begin{tabular}{|c|c|c|c|}
\hline $\begin{array}{c}\text { Interval } \\
\text { Number }\end{array}$ & $\begin{array}{c}\text { Minimun } \\
\text { Value }\end{array}$ & $\begin{array}{c}\text { Maximun } \\
\text { Value }\end{array}$ & $\begin{array}{c}\text { Assigned } \\
\text { Color }\end{array}$ \\
\hline 1 & 0 & $\min$ & Blue \\
\hline 2 & $\min +1$ & $\max$ & Royal Blue \\
\hline 3 & $\max +1$ & $\max +11$ & Green-Blue \\
\hline 4 & $\max +12$ & $\max +22$ & Green \\
\hline 5 & $\max +23$ & $\max +33$ & Yellow-Green \\
\hline 6 & $\max +34$ & 254 & Yellow \\
\hline 7 & 255 & 1024 & White \\
\hline
\end{tabular}

The proposed software eases the analysis of bone scan allowing the specialist to determine the presence or absence of metastatic disease, from the observation of the histograms along with a previous knowledge of thresholding or filtering techniques, without the necessity of directly observing the bone scan.

The intervals 3-5 are useful to illustrate the metastatic disease advance. The intervals 3 and 4 indicate the presence of early-stage metastasis, and intervals 5 and 6 an ongoing metastasis. The intervals used to indicate early-stage metastatic disease have and incremental step of 10 gray tones each. The rest of the intervals indicate the presence of advanced metastasis, with the gray tone value scaling with the metastasis stage. The latter three colors in Table 1 can be arbitrarily chosen based on the specialist requirements. Tones closer to yellow in the resulting image 
will indicate a more advanced stage of the metastatic disease in the ROI. Regions with metastatic disease as well as those with gray tones above 255 , corresponding to the bladder case or to cases where radiotracer was injected, will be displayed in white in the visualization tool.

\section{RESULTS AND DISCUSSION}

The previously shown Table 1 was obtained from the histogram analysis, considering its minimun and maximun values.

We apply the scheme in the table to three healthy skulls free of metastasis and we show the results in false color in Figure 3. In this case, the three images colors should be royal blue for the background and blue for the ROI. It should be noticed that as in the case of Figure 2d and 2f, the max values for both ROIs were not suitable since the three ROIs are free of metastasis. However, in this case, it did not alter the diagnosis. The tones problem could be corrected using the min and max values for each age, considering that the ranges in the table for each ROI as well as the corresponding analysis are dynamical, leaving room for a manual correction.

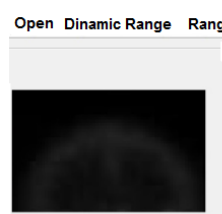

(a)

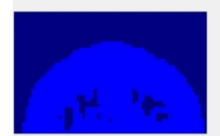

(d)

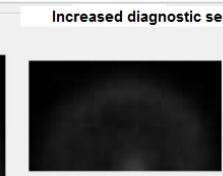

(b)

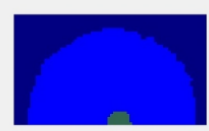

(e)

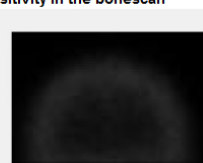

(c)

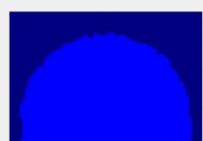

(f)

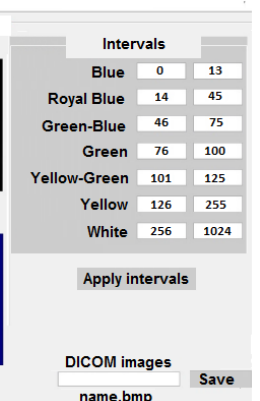

name.bmp
FIGURE 3. Application of the scheme shown in the table in the right panel of the figure for three healthy skulls.

In Figure 4 we show the results for three skulls with degenerative diseases. Figure $4 \mathrm{e}$ and $4 \mathrm{f}$ show and increase in the radiotracer absorption, with the extension and geometry of non-malignant diseases.

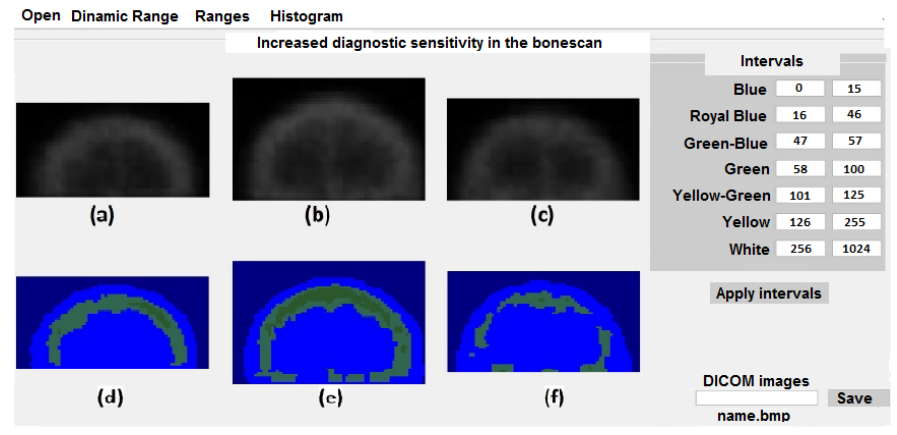

FIGURE 4. Application of the scheme shown in the table in the right panel of the figure for three skulls with bone degenerative diseases.

In Figure 5 we show the results for the pelvis case. We observe a region with high gray tone values, and two regions in black. The bladder tones were set to zero to ease the visualization of the ROI.

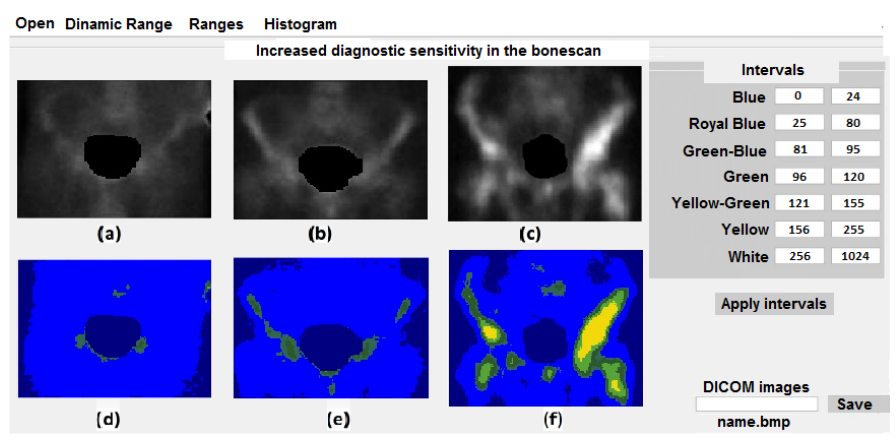

FIGURE 5. Application of the scheme shown in the table in the right panel of the figure for three pelvises.

In the case of a ROI free of metastasis but with a broadened histogram (Figures 1.c and 1.d), it is proposed to split the interval 2 of Table 1 in two extra sub-intervals in order to find the cause of the broadening and the advance of the disease. This division eases the observation of the degenerative diseases in the displayed ROI. This procedure is highly recommended in pelvis and skull cases, where the bones have the highest probability of generate degenerative diseases.

The image in the bottom left-most panel corresponds to a healthy pelvis. The image in the bottom middle panel illustrates the application of the comparison technique, 
thoroughly explained in a work developed by Jaramillo et $a l^{[8]}$, intended to assist the diagnosis procedure. It should be noticed that two regions of the pelvis have the same colors, corresponding to early stages of a bone degenerative disease, such as osteopenia or osteoporosis. These regions are shown in the right-most panels. If the differences are present only in one side, such a difference suggests the presence of a severe disease. Despite the similarities between the middle and right panels, the mild differences in the extension and geometry suggest different bone degenerative diseases in each case.

Finally, Figure 6 shows the histogram corresponding to a shoulder region. It could be noticed that the histogram values are irregular, hindering the visual acquisition of the min and max values. Hence, such values could be obtained only by applying the software.

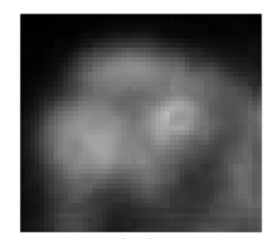

(a)

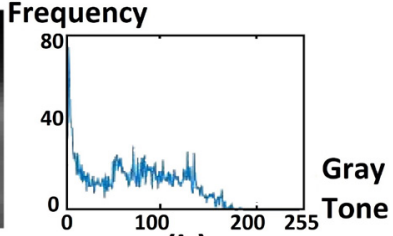

(b)
FIGURE 6. Histogram corresponding to a right shoulder.

\section{Discussion}

We recall that the gray tone values of a histogram corresponding to a patient free of bone metastatic disease are below 60. For a patient with an ongoing metastasis, the gray tones increase, being below 100, except in the case of advanced metastatic disease. Our choice of the 7-colors palette is then suitable for visualizing the bone scan in colors. The boundaries between colors observed in the ROIs can be related to the results obtained by applying edge detection techniques. Such techniques are widely-used in the segmentation of images. In this case, the edges correspond to the pixels with gray tones equal to the upper limits of the intervals described in the tables. Such limits could be regarded as level curves of a function $f(x, y)=$ cte corresponding to a ROI ${ }^{[12]}$.
As we have previously stressed, there are only two regions reaching the highest gray tone value (1024), the bladder and the region where the radiotracer was injected. The bladder stores the non-absorbed radiotracer, which is subsequently expelled through the urine. These two regions are not considered as regions of interest for diagnosis purposes. Hence, we set the gray tones of those regions to zero to ease the ROI visualization procedure, which can be observed in black in Figures 4 and 5 .

The gray tones of the bone scan images are low in general, yet considering the high bit depths, which are not fully exploited. Hence, early-stage metastatic disease cannot be diagnosed by directly observing an image, it is necessary to use the image gray tones histogram.

Our proposed method involves a simple technique with potential to be used in oncology centers requiring accuracy and experience. For example, a shift to the left of the min and max values of the histograms, would suggest the presence of bone anomaly in the ROI. The same results would be obtained from a broadened histogram. Additional gray tones observed along the $\mathrm{x}$-axis indicate the presence of metastatic diseases in the ROI. The advance of the metastasis is given in terms of the gray tones extension along the x-axis, i.e., the highest gray tone and the fraction of pixels reaching such a value.

This technique is particularly useful in oncology centers with large patients flow, or with limited resource due to its efficiency and execution times, allowing the specialists to diagnose more patients faster.

On the other hand, considering that a LUT table is built from a mathematical principle, with each element having an input value and assigned resulting output value, we classify table 1 as a LUT table. In nuclear medicine, LUT tables are built for each detec- 
tor as a function of the quantitative physical parameters, having several applications. In our particular case, such a table has only one application: converting input gray tones into an output colors. However, in general cases, for some intervals, for instance, 6 and 7, the input values are assigned without following any specific criterion, which is not the case of the LUT tables in Nuclear Medicine ${ }^{[13]}$.

\section{CONCLUSIONS}

In this work we introduced a method to analyze bone scans based on the visualization of a ROI gray levels histogram. We subsequently assigned false color to segmented regions in order to determine the advance of metastatic disease. The colors are assigned in terms of the max and min values of the observed histogram, with the last assignation intervals used for the metastasis detection and stage classification purposes only. Hence, such intervals are used only for visualization of the infiltrated regions, increasing their contrast, with the aim of comparing them with the healthy zones. This method allows a practical evaluation using the histogram of the image gray levels or the visualization of the ROI in color. The software interface is friendly. It is constituted by a straightforward Matlab GUI, with the potential to be used in oncology centers.

\section{AUTHOR CONTRIBUTIONS}

B.S. introduced the most specific Matlab algorithms, and performed the interpretation of mathematicalphysical models applied to the analysis of Scintigraphy. She also contributed to building the Matlab GUIs (Graphical User Interfaces) following the specifications of the other authors, that contains and execute all the developed algorithms, to optimize the operation for users unfamiliar with our proposal but with prior knowledge in bone cancer diagnosis from gamma scans. B.C. contributed to translating the paper to English language, she also contributed to the implementation of algorithms and their performance comparison in other programming languages such as Python and Fortran to improve our research. She also contributed to the GUIs development by optimizing the visualization tasks, as well as the comparison of the model of our proposal with other methods using image processing and analysis of medical images for diagnosis. M.P. the corresponding author, submitted the manuscript to the SOMIB platform and sent the requested information to the journal. She received the notifications and reviewer's reports. She kept the authors up to date regarding the manuscript's status and related journal protocols. Her support was important during the results visualization as well as in the last details of the GUI. A.J. the main researcher (P.I.)., has been working on this idea for several years and published papers with related results in the area. He is the liaison with the IMSS staff (Instituto Mexicano del Seguro Social-Mexican Social Security Institute), who were responsible for providing the gamma scans used in our work, following all the legal, safety and security protocols. He wrote the research protocol required for using the gramma scans and specialists' evaluations in the present research. J.A. contributed to editing and correcting the manuscript before submission to the journal and during the reviewing process. He actively contributed with A.J. in the construction of the mathematical physical model relating the histogram analysis, image processing, and filtering to build the used algorithms as well as the tables and figures preparation.

\section{ETHICAL STATEMENT}

Ethical Research Committee of IMSS approved and supervised the study complied with all applicable research and ethical standards and laws. 


\section{REFERENCES}

[1] McAninch J, Lue T. Smith and Tanagho's General Urology. 19th ed. EUA: The McGraw-Hill Companies, Inc; 2008. 351- 376p.

[2] Secretaria de Salud. Cáncer de próstata, padecimiento mortal y silencioso. Secretaría de Salud México [Internet]. 2017; Available from: https://www.gob.mx/salud/prensa/514-cancer-de-prostatapadecimiento-mortal-y-silencioso

[3] Coleman RE. Metastatic bone disease: clinical features, pathophysiology and treatment strategies. Cancer Treat Rev [Internet]. 2001;27(3):165-76. Available from: https://doi.org/10.1053/ctrv.2000.0210

[4] Zafeirakis A. Scoring systems of quantitative bone scanning in prostate cancer: historical overview, current status and future perspectives. Hell J Nucl Med [Internet]. 2014;17(2):136-144. Available from: https://doi.org/10.1967/s002449910134

[5] Koizumi M, Wagatsuma K, Miyaji N, et al. Evaluation of a computer-assisted diagnosis system, BONENAVI version 2, for bone scintigraphy in cancer patients in a routine clinical setting. Ann Nucl Med [Internet]. 2015;29(2):138-148. Available from: https://doi.org/10.1007/s12149-014-0921-y

[6] Sadik M, Suurkula M, Höglund P, Järund A, Edenbrandt L. Improved classifications of planar whole-body bone scans using a computer-assisted diagnosis system: a multicenter, multiplereader, multiple-case study. J Nucl Med [Internet]. 2009;50(3):368375. Available from: https://doi.org/10.2967/inumed.108.058883
[7] Pérez-Meza M, Jaramillo-Núñez A, Sánchez-Rinza BE. Visualizando Gammagramas Óseos en Colores. RMIB [Internet]. 2018;39(3):225237. Available from: https://doi.org/10.17488/rmib.39.3.2

[8] Jaramillo-Núñez A, Gómez-Conde JC. Método para incrementar la sensibilidad diagnóstica del gammagrama óseo. An Radiol Mex. 2015;14(1):11-19.

[9] Otsu NA. Threshold selection method from gray-level histograms. IEEE Trans Syst Man Cyb [Internet]. 1979;9(1):62-66. Available from: https://doi.org/10.1109/TSMC.1979.4310076

[10] Reddi SS, Rudin SF, Keshavan HK. An optimal multiple threshold scheme for image segmentation. IEEE Trans Syst Man Cyb [Internet]. 1984;SMC-14(4):661-665. Available from: https://doi.org/10.1109/TSIMC.1984.6313341

[11] Demirkaya O, Asyali MH, Sahoo PK. Image Processing with MATLAB: Applications in Medicine and Biology. EUA: CRC Press; 2009. 458p.

[12] Leithold L. El cálculo con geometría analítica. 6th ed. Distrito Federal: Harla; 1992. 1175-78p.

[13] Peinado MA. Monitores e impresoras. In Sociedad Española de Física Médica. Introducción al control de calidad en radiología. España: ADI: Librería/editorial científico-técnica; 2013. 147-148p. 